\title{
Diagnostic Value of Elevated D-Dimer Level in Venous Thromboembolism in Patients With Acute or Subacute Brain Lesions
}

\author{
Yeon Jin Kim, MD, Sun Im, MD, PhD, Yong Jun Jang, MD, \\ So Young Park, MD, Dong Gyun Sohn, MD, Geun-Young Park, MD, PhD
}

Department of Rehabilitation Medicine, College of Medicine, The Catholic University of Korea, Seoul, Korea

\begin{abstract}
Objective To define the risk factors that influence the occurrence of venous thromboembolism (VTE) in patients with acute or subacute brain lesions and to determine the usefulness of D-dimer levels for VTE screening of these patients.

Methods Medical data from January 2012 to December 2013 were retrospectively reviewed. Mean D-dimer levels in those with VTE versus those without VTE were compared. Factors associated with VTE were analyzed and the odds ratios (ORs) were calculated. The D-dimer cutoff value for patients with hemiplegia was defined using a receiver operating characteristic (ROC) curve.

Results Of 117 patients with acute or subacute brain lesions, 65 patients with elevated D-dimer levels (mean, $5.1 \pm 5.8 \mathrm{mg} / \mathrm{L}$; positive result $>0.55 \mathrm{mg} / \mathrm{L}$ ) were identified. Logistic regression analysis showed that the risk of VTE was 3.9 times higher in those with urinary tract infections (UTIs) ( $\mathrm{p}=0.0255$ ). The risk of VTE was 4.5 times higher in those who had recently undergone surgery $(\mathrm{p}=0.0151)$. Analysis of the ROC showed $3.95 \mathrm{mg} / \mathrm{L}$ to be the appropriate D-dimer cutoff value for screening for VTE (area under the curve [AUC], 0.63; 95\% confidence interval [CI], 0.5-0.8) in patients with acute or subacute brain lesions. This differs greatly from the conventional D-dimer cutoff value of $0.55 \mathrm{mg} / \mathrm{L}$. D-dimer levels less than $3.95 \mathrm{mg} / \mathrm{L}$ in the absence of surgery showed a negative predictive value of $95.8 \%$ (95\% CI, 78.8-99.8).

Conclusion Elevated D-dimer levels alone have some value in VTE diagnosis. However, the concomitant presence of UTI or a history of recent surgery significantly increased the risk of VTE in patients with acute or subacute brain lesions. Therefore, a different D-dimer cutoff value should be applied in these cases.
\end{abstract}

Keywords Brain diseases, Fibrin fragment D, Venous thrombosis, Pulmonary embolism

Received April 20, 2015; Accepted July 1, 2015

Corresponding author: Geun-Young Park

Department of Rehabilitation Medicine, Bucheon St. Mary's Hospital, College of Medicine, The Catholic University of Korea, 327 Sosa-ro, Wonmi-gu, Bucheon 14647, Korea

Tel: +82-32-340-2182, Fax: +82-32-340-7640, E-mail: rootpmr@catholic.ac.kr

() This is an open-access article distributed under the terms of the Creative Commons Attribution Non-Commercial License (http://creativecommons.org/ licenses/by-nc/4.0) which permits unrestricted noncommercial use, distribution, and reproduction in any medium, provided the original work is properly cited. Copyright $\odot 2015$ by Korean Academy of Rehabilitation Medicine 


\section{INTRODUCTION}

Deep vein thrombosis (DVT) and pulmonary embolism (PE), two manifestations of venous thromboembolism (VTE), are common complications in patients with disabilities undergoing rehabilitation. The reported incidence of VTE varies widely, depending on the study, but the average is $40 \%-60 \%$ for DVT and $10 \%$ for PE in stroke patients [1]. The incidence of DVT in patients with major traumatic brain injury (TBI) is 54\% [2]. Although DVT and PE typically cause symptoms such as leg swelling, tenderness, or redness, in some cases, they become quite severe without showing any discernable signs or symptoms. Therefore, it is crucial to be able to screen for these conditions physiologically. A D-dimer test is noninvasive, relatively easy to perform, and provides fast results. Therefore, it provides a useful tool for screening for VTE. Regarding VTE diagnosis, D-dimer assays are known to show high sensitivity (93\%-95\%) but relatively low specificity (40\%-60\%) [3-5]. Plasma levels of D-dimer are increased in the presence of thrombotic diseases. Unfortunately, D-dimer levels are also increased in other conditions, such as senescence, recent surgery, stroke, trauma, infection, malignancy, acute coronary disease, heart failure, chronic renal disease, liver disease, elevated rheumatoid factors, and pregnancy $[3,6,7]$.

Patients with brain lesions have elevated basal D-dimer levels. Matsumoto et al. [8] and Park et al. [9] proposed that when infarction volume increases, the level of plasma D-dimer also increases. Prell et al. [10] revealed that D-dimer levels are elevated after craniotomy. Therefore, use of the conventional D-dimer cutoff value when evaluating these patients can be confounded by the presence of brain lesions. The purpose of this study was to define the appropriate D-dimer cutoff value for screening for VTE in patients with acute or subacute brain lesions. In addition, we aimed to determine clinical factors that can influence the risk of VTE development in the presence of elevated D-dimer levels.

\section{MATERIALS AND METHODS}

\section{Subjects}

A retrospective study was performed by reviewing medical charts of 455 inpatients who had been admitted to the rehabilitation department at our institution between
Admission patients due to brain lesion Jan 2012-Dec 2013

$\mathrm{n}=455$

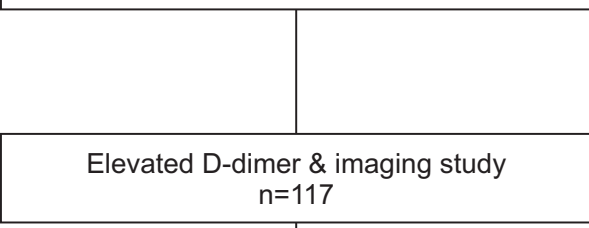

Eligible cases satisfying inclusion driteria $\mathrm{n}=65$

Patients with venous thromboembolism confirmed from imaging study, $n=21$

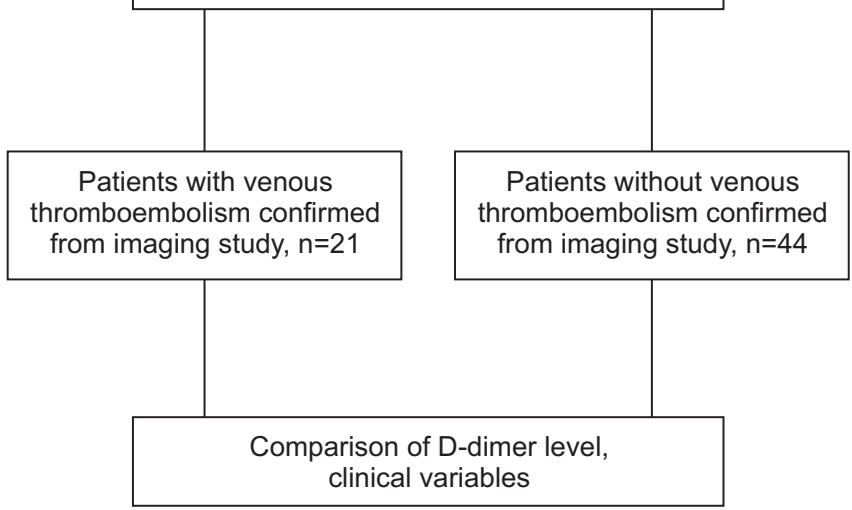

Exclusion criteria

- Recurrent cerebral vascular

accident, $\mathrm{n}=35$

- Anti-platelet agents, $n=9$

- In premorbid gait disturbance, $n=8$
Fig. 1. Flow chart showing the steps used for identifying the study cohort. 
January 20, 2012 and December 30, 2013. Of those 455 patients, 117 underwent imaging studies, such as DVT computed tomography (CT), pulmonary angio CT, or lower extremity duplex sonography, because of elevations in Ddimer levels. Patients with recurrent brain lesions, those who were unable to walk independently, those who took anticoagulants or anti-platelet agents prior to admission, and those who had blood coagulation disorders were excluded from the study (Fig. 1).

\section{Methods}

The following parameters were recorded: gender, age, preexisting medical conditions, elapsed time from disease onset, neurologic and physical examination results, blood test and urinary analysis, and results of imaging studies. Among various factors known to affect VTE risk, gender, brain lesion, recent surgery, presence of infections such as urinary tract infections (UTIs) and pneumonia, Medical Research Council (MRC) scale, results of the Korean version of the Mini-Mental State Examination (MMSE-K), and results of the Korean version of the Modified Barthel Index (K-MBI) were evaluated [11-14]. To define each category, brain lesion was grouped by etiology (infarction, hemorrhage, or TBI). Recent surgery was defined as surgeries or interventions through blood vessels which were performed within 100 days from the date of the D-dimer test $[15,16]$. UTI was identified in those patients positive for pyuria ( $>8$ leukocytes per high-power field) or bacteriuria (positive nitrite or $>1$ organism per oil-immersion field) from urinalysis obtained within a week of the date of the D-dimer test. Pneumonia was confirmed by radiologists through chest radiography or chest computed tomography. The degree of motor weakness was assessed using the MRC scale at the time of transfer to our department. MRC score $<3$ was categorized as 'severe weakness', and MRC score $\geq 3$ in all extremities was categorized as 'mild weakness'. Cognitive function was evaluated using the MMSE-K $[11,12]$ and clinically grouped into 3 grades: 'normal' (score $\geq 24$ ), 'suspicion of dementia' (score 20-23), and 'definite dementia' (score $<20$ ). We defined MMSE-K score $<24$ as 'cognitive dysfunction' and MMSE-K score $\geq 24$ as 'normal cognition' [11,12]. Activity was evaluated using K-MBI, which was measured by occupational therapists. Brain lesion disability was classified as follows: K-MBI $\leq 24$ is class 1 , indicating the most severe disability. Those pa- tients with K-MBI scores $>24$ were classified as ' minimally to moderately dependent' [13]. We divided enrolled patients into groups A and B. Group A included patients with VTE and group B was comprised of patients without VTE. The differences between groups A and B were analyzed.

To measure D-dimer levels, a venous blood sample was placed into a test tube containing sodium citrate, and a centrifugation latex assay was done (Latex Test BL2P-FDP; Biolinks Co. Ltd., Tokyo, Japan). D-dimer concentration $>0.55 \mathrm{mg} / \mathrm{L}$ was considered a positive result. For those who had undergone multiple D-dimer evaluations, the last value obtained before imaging was used for analysis. For those with non-elevated D-dimer levels and those who did not receive a diagnosis of VTE during the study period, medical charts from 1-2 years after the study period were examined for the development of VTE.

Univariate and multivariate logistic regression analyses were performed to identify factors associated with VTE. Using a receiver operator characteristic (ROC) curve, an adjusted D-dimer cutoff value for patients with brain lesions was defined.

\section{Statistical analysis}

Descriptive statistics are presented as counts and percentages for categorical variables and mean \pm standard deviation (SD) for continuous variables. A comparison of categorical variables was performed using chi-square test and Fisher exact test. Univariate and multivariate logistic regressions were performed to identify independent predictors of VTE. The sensitivity, specificity, accuracy, positive predictive value (PPV), and negative predictive value (NPV) of D-dimer levels were calculated with respect to VTE. Diagnostic performance was compared using a chisquare test and Fisher exact test. Values of $p<0.05$ were considered statistically significant. Statistical analyses were performed with SAS Enterprise Guide 5.1 for Windows (SAS Institute, Cary, NC, USA).

\section{RESULTS}

A total of 65 patients met the inclusion criteria. Among them, 21 patients were diagnosed with VTE via imaging studies. The incidence of VTE in patients with brain lesions admitted to our rehabilitation unit was $4.7 \%$. Average age, time from disease onset, and D-dimer levels are 
Table 1. Baseline characteristics of enrolled patients showing the association between baseline characteristics and incidence of VTE

\begin{tabular}{|c|c|c|c|c|}
\hline & Total $(n=65)$ & Group A $(n=21)$ & Group B $(n=44)$ & p-value \\
\hline Age (yr) & $65.7 \pm 14.2$ & $65.8 \pm 15.2$ & $65.7 \pm 13.9$ & 0.872 \\
\hline \multicolumn{5}{|l|}{ Gender } \\
\hline Male & $36(55.4)$ & $9(42.9)$ & $27(61.4)$ & 0.160 \\
\hline Female & $29(44.6)$ & $12(57.1)$ & $17(38.6)$ & \\
\hline Length of elapsed time (day) & $27.0 \pm 36.3$ & $34.0 \pm 56.9$ & $23.7 \pm 20.6$ & 0.225 \\
\hline \multicolumn{5}{|l|}{ Brain lesion } \\
\hline Infarction & $26(40.0)$ & $7(33.3)$ & $20(45.5)$ & 0.354 \\
\hline ICH & $26(40.0)$ & $8(38.1)$ & $19(43.2)$ & 0.697 \\
\hline TBI & $13(20.0)$ & $6(28.6)$ & $9(20.5)$ & 0.535 \\
\hline \multicolumn{5}{|l|}{ Recent surgery } \\
\hline Present & $31(47.7)$ & $15(71.4)$ & $16(36.4)$ & $0.008^{*}$ \\
\hline Absent & $34(52.3)$ & $6(28.6)$ & $28(63.6)$ & \\
\hline \multicolumn{5}{|l|}{ Pneumonia } \\
\hline Present & $16(24.6)$ & $4(19.0)$ & $12(27.3)$ & 0.472 \\
\hline Absent & $49(75.4)$ & $17(81.0)$ & $32(72.7)$ & \\
\hline \multicolumn{5}{|l|}{ UTI } \\
\hline Present & $30(46.2)$ & $14(66.7)$ & $16(36.4)$ & $0.022^{*}$ \\
\hline Absent & $35(53.8)$ & $7(33.3)$ & $28(63.6)$ & \\
\hline \multicolumn{5}{|l|}{ MRC scale } \\
\hline $0-2$ & $53(81.5)$ & $18(85.7)$ & $35(79.5)$ & 0.737 \\
\hline $3-5$ & $12(18.5)$ & $3(14.3)$ & $9(20.5)$ & \\
\hline MMSE-K & $7.9 \pm 9.7$ & $6.1 \pm 7.9$ & $8.8 \pm 10.4$ & 0.434 \\
\hline$<24$ & $57(87.7)$ & $20(95.2)$ & $37(84.1)$ & 0.259 \\
\hline$\geq 24$ & $8(12.3)$ & $1(4.8)$ & $7(15.9)$ & \\
\hline K-MBI & $11.1 \pm 23.7$ & $7.0 \pm 20.4$ & $13.1 \pm 25.2$ & 0.287 \\
\hline$\leq 24$ & $54(83.1)$ & $19(90.5)$ & $35(79.5)$ & 0.480 \\
\hline$>24$ & $11(16.9)$ & $2(9.5)$ & $9(20.5)$ & \\
\hline D-dimer level (mg/L) & $5.1 \pm 5.8$ & $6.8 \pm 7.7$ & $4.2 \pm 4.5$ & 0.088 \\
\hline
\end{tabular}

Values are presented as mean \pm standard deviation or number (\%).

VTE, venous thromboembolism; ICH, intra-cranial hemorrhage; TBI, traumatic brain injury; UTI, urinary tract infection; MRC, Medical Research Council; MMSE-K, Korean version of the Mini-Mental State Examination; K-MBI, Korean version of the Modified Barthel Index.

The p-values were calculated using the chi-square test and Fisher exact test. Group A consisted of patients with VTE and group B consisted of patients without VTE.

${ }^{*} \mathrm{p}<0.05$.

shown in Table 1, and divided by demographic features. Among the 65 patients, atrial fibrillation was diagnosed in 2 patients, one of whom had non-valvular atrial fibrillation.

The mean D-dimer level in all patients was 5.1 (SD 5.8; range 0.7-35.2). Mean D-dimer levels for group A and group B were 6.8 (SD 7.7; range 1.4-35.2) and 4.2 (SD 4.5; range $0.7-27.7)$, respectively ( $\mathrm{p}=0.088)$. Comparison of baseline characteristics and clinical features are shown in Tables 1 and 2. No one in the control group or with a low D-dimer level who was admitted during the study period had any medical record of VTE in the 2-year follow-up.

In univariate logistic regression analysis, a history of recent surgery or the presence of a UTI was significantly 
Table 2. Association between baseline characteristics and D-dimer levels

\begin{tabular}{|c|c|c|c|c|}
\hline \multirow{2}{*}{ D-dimer } & \multicolumn{2}{|c|}{ Group A $(n=21)$} & \multicolumn{2}{|c|}{ Group B $(n=44)$} \\
\hline & Mean (SD) & p-value & Mean (SD) & p-value \\
\hline \multicolumn{5}{|l|}{ Gender } \\
\hline Male & $4.8(3.2)$ & 0.500 & $4.4(5.5)$ & 0.268 \\
\hline Female & $8.3(9.6)$ & & $4.0(2.0)$ & \\
\hline \multicolumn{5}{|l|}{ Brain lesion } \\
\hline Infarction & $4.5(1.9)$ & 0.737 & $4.4(5.7)$ & 0.741 \\
\hline $\mathrm{ICH}$ & $10.3(11.6)$ & 0.538 & $4.0(3.0)$ & 0.972 \\
\hline TBI & $4.9(3.4)$ & 0.785 & $3.6(3.5)$ & 0.399 \\
\hline \multicolumn{5}{|c|}{ Recent surgery } \\
\hline Present & $7.1(8.5)$ & 0.907 & $3.8(4.9)$ & 0.140 \\
\hline Absent & $6.1(5.7)$ & & $4.9(3.6)$ & \\
\hline \multicolumn{5}{|l|}{ Pneumonia } \\
\hline Present & $4.0(1.8)$ & 0.622 & $4.5(4.9)$ & 0.257 \\
\hline Absent & $7.5(8.4)$ & & $3.5(3.2)$ & \\
\hline \multicolumn{5}{|l|}{ UTI } \\
\hline Present & $7.3(8.9)$ & 0.852 & $4.5(5.1)$ & 0.464 \\
\hline Absent & $5.9(4.6)$ & & $3.8(3.1)$ & \\
\hline \multicolumn{5}{|l|}{ MRC scale } \\
\hline $0-2$ & $6.9(8.1)$ & 0.960 & $4.5(4.9)$ & 0.532 \\
\hline $3-5$ & $6.3(5.2)$ & & $3.1(2.0)$ & \\
\hline \multicolumn{5}{|l|}{ MMSE-K } \\
\hline$<24$ & $7.0(7.8)$ & 0.804 & $3.8(2.8)$ & 0.653 \\
\hline$\geq 24$ & $4.0(-)$ & & $6.5(9.4)$ & \\
\hline \multicolumn{5}{|l|}{ K-MBI } \\
\hline$\leq 24$ & $6.7(7.9)$ & 0.510 & $4.6(4.9)$ & 0.600 \\
\hline$>24$ & $8.0(5.8)$ & & $2.8(1.4)$ & \\
\hline
\end{tabular}

SD, standard deviation; ICH, intra-cranial hemorrhage; TBI, traumatic brain injury; UTI, urinary tract infection; MRC, Medical Research Council; MMSE-K, Korean version of the Mini-Mental State Examination; K-MBI, Korean version of the Modified Barthel Index; VTE, venous thromboembolism.

Statistics were carried out using the Wilcoxon signedrank test. Group A consisted of patients with VTE and group B consisted of patients without VTE.

associated with VTE $(\mathrm{p}<0.05)$. In multivariate logistic regression, odds ratios of patients who had recently undergone surgery and patients with UTIs were 4.5 (95\% confidence interval [CI], 1.3-15.0; $\mathrm{p}=0.0151)$ and $3.9(95 \%$ CI, 1.2-13.1; $\mathrm{p}=0.026$ ), respectively (Table 3).

The ROC curve revealed that the adjusted D-dimer cutoff value for patients with brain lesions was $3.95 \mathrm{mg} / \mathrm{L}$. An area under the curve (AUC) of $>0.9$ was considered 'excellent', 0.8-0.9 'very good', 0.7-0.8 'good', 0.6-0.7 'average', and $<0.6$ 'poor' [17]. When we applied the adjusted Ddimer cutoff value of $3.95 \mathrm{mg} / \mathrm{L}$, AUC was 0.63 (average), sensitivity was $61.9 \%$, and specificity was $70.5 \%$ (Fig. 2). When we applied the conventional D-dimer cutoff value of $0.55 \mathrm{mg} / \mathrm{L}$, specificity was not measurable. For UTI patients, D-dimer levels of $>3.52 \mathrm{mg} / \mathrm{L}$ had increased PPV and AUC. For patients who had not recently undergone surgery, D-dimer levels of $\leq 3.95 \mathrm{mg} / \mathrm{L}$ showed increased sensitivity, specificity, and NPV up to $95.8 \%$ (Table 4).

\section{DISCUSSION}

The results of this study showed that applying the conventional D-dimer cutoff value $(>0.55 \mathrm{mg} / \mathrm{L})$ in patients with brain lesions has poor value in VTE diagnosis. Our results reveal that a more useful cutoff value is $3.95 \mathrm{mg} / \mathrm{L}$ in patients with acute or subacute brain lesions. Patients with brain lesions and concomitant UTIs or recent surgical history had an increased risk of VTE (3.9- and 4.5-fold higher, respectively). Therefore, in the presence of UTI, the cutoff value should be more strict ( $>3.52 \mathrm{mg} / \mathrm{L}$ ). In patients who had not recently undergone surgery, a Ddimer level $\leq 3.95 \mathrm{mg} / \mathrm{L}$ had a NPV for VTE of $95.8 \%$.

Chua et al. [18] found that the incidence of DVT in patients in a Singapore neuro-rehabilitation unit was 5.01\%. In contrast, Sachdev et al. [19], Yablon et al. [20], and Cifu et al. [21] reported incidences of DVT among inpatients in rehabilitation clinics of $34 \%, 11.1 \%$, and $11 \%$, respectively. According to our study, VTE incidence (including DVT) for patients with brain lesions who underwent rehabilitation treatment for 1 month was about $4.7 \%$, which was similar to that Chua et al.'s findings [18]. These discrepancies may be attributable to differences in ethnicity, age, severity of stroke, severity of motor weakness, and use of DVT prophylaxis.

Many studies have identified conditions that increase D-dimer levels. We found that UTI increased the risk of VTE 3.9-fold ( $\mathrm{p}=0.030$ ). Many studies have already shown a relationship between VTE and infection. Smeeth et al. [22] reported that UTI increased DVT and PE incidence up to 2.10- and 2.11-fold, respectively. Our results are in accordance with previous studies that found that UTI may increase the incidence of VTE through increased hypercoagulation $[22,23]$. Pneumonia is known to increase 
Table 3. Univariate and multivariate logistic regression analyses to identify factors associated with VTE

\begin{tabular}{|c|c|c|c|c|}
\hline & \multicolumn{2}{|c|}{ Univariate } & \multicolumn{2}{|c|}{ Multivariate } \\
\hline & OR (95\% CI) & p-value & OR $(95 \% \mathrm{CI})$ & p-value \\
\hline \multicolumn{5}{|l|}{ Gender } \\
\hline Male & 1 & & & \\
\hline Female & $2.1(0.7-6.1)$ & 0.164 & & \\
\hline \multicolumn{5}{|l|}{ Brain lesion } \\
\hline Infarction & $0.6(0.2-1.8)$ & 0.356 & & \\
\hline $\mathrm{ICH}$ & $0.6(0.2-1.8)$ & 0.356 & & \\
\hline TBI & $0.8(0.3-2.4)$ & 0.697 & & \\
\hline \multicolumn{5}{|l|}{ Recent surgery } \\
\hline Present & $4.4(1.4-13.5)$ & $0.010^{*}$ & $4.5(1.3-15.0)$ & $0.015^{*}$ \\
\hline Absent & 1 & & & \\
\hline \multicolumn{5}{|l|}{ UTI } \\
\hline Present & $3.5(1.2-10.5)$ & $0.025^{*}$ & $3.9(1.2-13.1)$ & $0.026^{*}$ \\
\hline Absent & 1 & & & \\
\hline \multicolumn{5}{|l|}{ Pneumonia } \\
\hline Present & $0.6(0.2-2.3)$ & 0.474 & & \\
\hline Absent & 1 & & & \\
\hline \multicolumn{5}{|l|}{ MRC scale } \\
\hline Severe weakness & $1.5(0.4-6.4)$ & 0.551 & & \\
\hline Mild weakness & 1 & & & \\
\hline \multicolumn{5}{|l|}{ Cognitive function } \\
\hline Cognitive dysfunction & $3.8(0.4-33.0)$ & 0.228 & & \\
\hline Normal cognition & 1 & & & \\
\hline \multicolumn{5}{|l|}{ K-MBI } \\
\hline Total dependent & $2.4(0.5-12.5)$ & 0.283 & & \\
\hline Mild to moderate dependent & 1 & & & \\
\hline
\end{tabular}

VTE, venous thromboembolism; OR, odds ratio; CI, confidence interval; ICH, intra-cranial hemorrhage; TBI, traumatic brain injury; UTI, urinary tract infection; MRC, Medical Research Council; K-MBI, Korean version of the Modified Barthel Index.

${ }^{*} \mathrm{p}<0.05$.

the incidence of VTE [22]. However, in our study, pneumonia had no significant relationship with the occurrence of VTE. This may be because there were fewer cases of pneumonia than UTI in this study, whereas previous reports recorded similar incidences of UTI and pneumonia. Because of the limited number of pneumonia patients in this study, further studies need to be done to elucidate what, if any, D-dimer cutoff level would be useful in diagnosing VTE in patients with pneumonia. In addition, the absence of recent surgery showed significant NPV in VTE diagnosis. This is in accordance with previous studies that found that surgical procedures increase the risk of VTE. Orthopedic surgery and vascular surgery increase VTE incidence, as reported in many previous studies $[6,7,15]$. Fujita el al. [24] stated that total or partial knee arthroplasty increased the incidence of VTE, whereas Kimmell and Jahromi [25] reported that craniotomy had a similar effect. The mechanism by which surgeries lead to VTE is unknown, but Kim et al. [26] postulated that an increase in the duration of an operation increases the risk of VTE. Putting those studies together, we posit that operation under general anesthesia in a stationary position might increase the risk of VTE in patients with brain lesions. Further studies are warranted in order to define the connection between long operations and VTE. Legnani et al. [27] and Pulivarthi and Gurram [28] re- 
ported that certain populations, such as the elderly and patients with cancer, might have elevated D-dimer levels. Therefore, they recommended that a different $\mathrm{D}$-dimer cutoff value should be applied in the elderly or patients with cancer. Many studies reported that D-dimer levels are elevated in cases of brain lesions, such as ischemic stroke, hemorrhage, and TBI $[9,29]$. Thus, in screening for VTE, the diagnostic value of an increase in D-dimer levels can be confounded by the presence of brain lesions. Indeed, the ROC curve of the 65 patients with brain lesions in our study showed that $3.95 \mathrm{mg} / \mathrm{L}$ was the ideal adjusted D-dimer cutoff value; however, the AUC had only an average discriminative value. In the presence of UTI, when a D-dimer cutoff value of $>3.52 \mathrm{mg} / \mathrm{L}$ was applied, the PPV was higher than when it was set at $>3.95$ $\mathrm{mg} / \mathrm{L}$. Thus, we postulate that a different $\mathrm{D}$-dimer cutoff

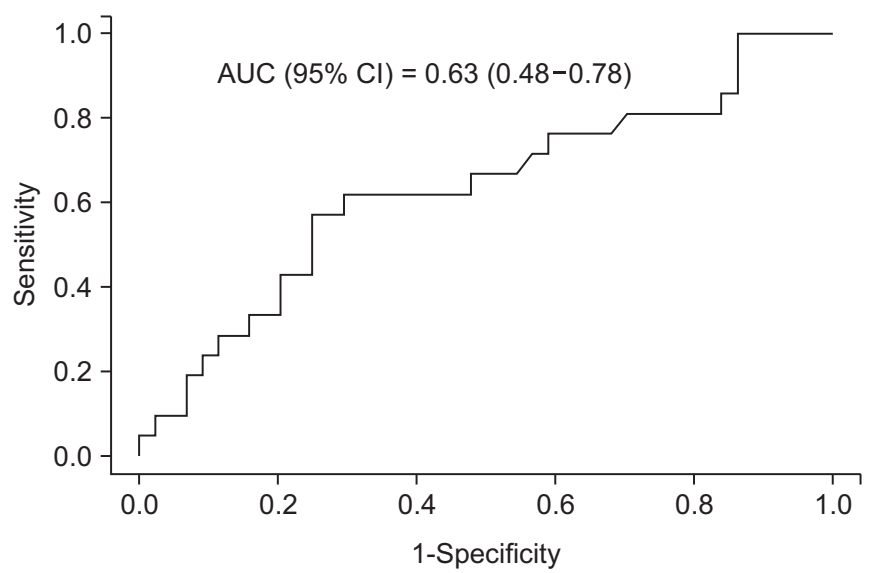

Fig. 2. Receiver operating characteristic (ROC) curve showing sensitivity and specificity of a D-dimer cutoff value of 3.95. AUC, area under the curve; $\mathrm{CI}$, confidence interval. value should be used for patients with UTI. Moreover, for patients who had not recently undergone surgery, D-dimer levels $\leq 3.95 \mathrm{mg} / \mathrm{L}$ were associated with a much lower incidence of VTE. Therefore, our study shows that in patients with brain lesions and elevated D-dimer levels, the presence of UTI or recent surgical history are important clinical factors to consider in the diagnosis of VTE.

Raising the level of D-dimer cutoff values for all patients may mistakenly exclude those with VTE but with low or normal D-dimer levels. However, we examined the medical charts of our subjects for 1-2 years after our study, and no one in the control group was readmitted to our hospital because of previously undiagnosed VTE. The cutoff value proposed here may vary depending on the method of D-dimer assessment [28]. Although the latex assay method could have theoretically missed those with false negative results, as shown in Table 3, the probability of a person who had not undergone a recent surgical procedure with a D-dimer level less than $3.95 \mathrm{mg} / \mathrm{L}$ being diagnosed with VTE was very low, with a NPV of $95.8 \%$. The findings of this study are significant as they imply that a different D-dimer cutoff value should be used in screening for VTE in patients admitted in a rehabilitation setting. Whether the particular cutoff value defined in this study could be used as a guideline in other clinical settings with different $\mathrm{D}$-dimer assessment methods is a topic that warrants further study, but the results of this study emphasize that along with elevated D-dimer levels, UTI and surgical procedures are important clinical factors that may affect the diagnosis of VTE in patients in a rehabilitation setting.

There were some limitations to this study. First, the study population mainly consisted of stroke and TBI

Table 4. Diagnostic performance of VTE screening in patients with brain lesions

\begin{tabular}{lccc}
\hline & Total $(\mathbf{n}=\mathbf{6 5})$ & UTI $(\mathbf{n}=\mathbf{3 0})$ & Absence of surgery $(\mathbf{n}=\mathbf{3 4})$ \\
\hline D-dimer level $(\mathrm{mg} / \mathrm{L})$ & $>3.95$ & $>3.52$ & $\leq 3.95$ \\
\hline AUC & $0.63(0.5-0.8)$ & $0.67(0.5-0.9)$ & $0.76(0.5-1.0)$ \\
Sensitivity & $61.9(38.4-81.9)$ & $64.3(35.1-87.2)$ & $83.3(35.9-99.6)$ \\
Specificity & $70.5(54.8-83.2)$ & $75.0(47.6-92.7)$ & $82.1(63.1-96.9)$ \\
PPV & $50.0(29.9-70.1)$ & $69.2(38.6-90.9)$ & $50.0(18.7-81.3)$ \\
NPV & $79.5(63.5-90.7)$ & $70.6(44.0-89.7)$ & $95.8(78.9-99.9)$ \\
Accuracy & $67.7(55.0-78.8)$ & $70.0(50.6-85.3)$ & $82.4(65.5-93.2)$ \\
\hline
\end{tabular}

Values are presented as $95 \%$ confidence interval.

VTE, venous thromboembolism; UTI, urinary tract infection; AUC, area under the curve; PPV, positive predictive value; NPV, negative predictive value. 
patients with severe neurologic deficits who were admitted to our rehabilitation department at the acute stage of treatment. The results may not be applicable to brain lesion patients with increased mobility and gait function or to chronic brain lesion patients. Second, clinical signs such as swelling or Homan sign could not be routinely evaluated and these factors were not analyzed in our study. However, patients with brain lesions may show only subtle or no symptoms, and physical examination may not always be reliable enough to confirm or exclude VTE [30]. Third, patients with normal D-dimer levels were not included in this study, which might affect the cutoff value. Fourth, this was a retrospective study performed mainly by reviewing medical charts, so the results should be confirmed in large-cohort studies. Fifth, we considered asymptomatic and symptomatic UTI as one homogeneous group and could not confirm whether the results differed between those with symptomatic versus asymptomatic UTI. Sixth, the reference standard suggested in our study might vary when using different D-dimer level assays. Seventh, we did not consider the possibility of false negative results when using the latex assay or whether adjusting the cutoff value could lead to an increased incidence of false negative results. Finally, certain brain lesions are known to affect the incidence of VTE. In our study, the type of brain lesion did not seem to affect the risk of VTE development, but due to the limited number of patients further prospective studies with larger cohorts need to be carried out to conclude whether certain types of brain lesions preferentially affect VTE development.

In conclusion, this study suggested that a D-dimer cutoff value for stroke and TBI patients should be set at $3.95 \mathrm{mg} /$ $\mathrm{L}$, or $>3.52 \mathrm{mg} / \mathrm{L}$ for patients with UTI, in screening for VTE. In addition, patients with D-dimer levels $\leq 3.95 \mathrm{mg} / \mathrm{L}$ who have not recently undergone surgery are at very low risk for VTE. Overall, our results showed that a higher Ddimer cutoff value than is conventionally applied should be considered for VTE diagnosis in patients with acute or subacute brain lesions in a rehabilitation setting.

\section{CONFLICT OF INTEREST}

No potential conflict of interest relevant to this article was reported.

\section{ACKNOWLEGEDMENTS}

The statistical consultation was supported by a grant of the Korean Health Technology R\&D Project, Ministry of Health \& Welfare, Republic of Korea (No. HI14C1731).

\section{REFERENCES}

1. Brandstater ME, Roth EJ, Siebens HC. Venous thromboembolism in stroke: literature review and implications for clinical practice. Arch Phys Med Rehabil 1992;73(5-S):S379-91.

2. Geerts WH, Code KI, Jay RM, Chen E, Szalai JP. A prospective study of venous thromboembolism after major trauma. N Engl J Med 1994;331:1601-6.

3. Hong MY, Lee C, Yoo SY, Shin DH, Cheong SS, Kwon $\mathrm{JH}$, et al. Cut-off value and factors associated with a false positive D-dimer result for venous thromboembolism in Koreans. Korean J Med 2013;84:372-8.

4. Schrecengost JE, LeGallo RD, Boyd JC, Moons KG, Gonias SL, Rose CE Jr, et al. Comparison of diagnostic accuracies in outpatients and hospitalized patients of D-dimer testing for the evaluation of suspected pulmonary embolism. Clin Chem 2003;49:1483-90.

5. Sadovsky R. ELISA D-dimer testing: high sensitivity but low specificity. Am Fam Physician 2002;66:2310.

6. Darwood RJ, Smith FC. Deep vein thrombosis. Surgery (Oxford) 2013;31:206-10.

7. Jiang Y, Li J, Liu Y, Li YC, Zhang WG. Risk factors for deep vein thrombosis after orthopedic surgery and the diagnostic value of D-dimer. Ann Vasc Surg 2015;29:675-81.

8. Matsumoto M, Sakaguchi M, Okazaki S, Furukado S, Tagaya M, Etani H, et al. Relationship between plasma (D)-dimer level and cerebral infarction volume in patients with nonvalvular atrial fibrillation. Cerebrovasc Dis 2013;35:64-72.

9. Park YW, Koh EJ, Choi HY. Correlation between serum D-dimer level and volume in acute Ischemic stroke. J Korean Neurosurg Soc 2011;50:89-94.

10. Prell J, Rachinger J, Smaczny R, Taute BM, Rampp S, Illert J, et al. D-dimer plasma level: a reliable marker for venous thromboembolism after elective craniotomy. J Neurosurg 2013;119:1340-6.

11. Park JH, Kwon YC. Standardization of Korean version of the Mini-Mental State Examination (MMSE-K) for 
use in the elderly. Part II: diagnostic validity. J Korean Neuropsychiatr Assoc 1989;28:508-13.

12. Lee HJ, Choi HY, Yun KW, Kim YC, Lim WJ, Kim JH, et al. Association between obesity indices and MMSE-K in elderly. J Korean Neuropsychiatr Assoc 2013;52:44753.

13. Jung HY, Park BK, Shin HS, Kang YK, Pyun SB, Paik NJ, et al. Development of the Korean version of Modified Barthel Index (K-MBI): multi-center study for subjects with stroke. J Korean Acad Rehabil Med 2007;31:28397.

14. Kim EJ, Kim SS, Kim WH, Lee WJ, Nam KY, Park CW, et al. The selection of exercise stress test in hemiplegic patients. J Korean Acad Rehabil Med 2008;32:2631.

15. Ramanan B, Gupta PK, Sundaram A, Lynch TG, MacTaggart JN, Baxter BT, et al. In-hospital and postdischarge venous thromboembolism after vascular surgery. J Vasc Surg 2013;57:1589-96.

16. Jeong HS, Miller TJ, Davis K, Matthew A, Lysikowski J, Lazcano E, et al. Application of the Caprini risk assessment model in evaluation of non-venous thromboembolism complications in plastic and reconstructive surgery patients. Aesthet Surg J 2014;34:87-95

17. Cho KH, Kim KP, Woo BC, Kim YJ, Park JY, Cho SY, et al. Relationship between blood stasis syndrome score and cardioankle vascular index in stroke patients. Evid Based Complement Alternat Med 2012;2012:696983.

18. Chua K, Kong KH, Chan SP. Prevalence and risk factors of asymptomatic lower extremity deep venous thrombosis in Asian neurorehabilitation admissions in Singapore. Arch Phys Med Rehabil 2008;89:2316-23.

19. Sachdev U, Teodorescu VJ, Shao M, Russo T, Jacobs TS, Silverberg D, et al. Incidence and distribution of lower extremity deep vein thrombosis in rehabilitation patients: implications for screening. Vasc Endovascular Surg 2006;40:205-11.

20. Yablon SA, Rock WA Jr, Nick TG, Sherer M, McGrath $\mathrm{CM}$, Goodson KH. Deep vein thrombosis: prevalence and risk factors in rehabilitation admissions with brain injury. Neurology 2004;63:485-91.

21. Cifu DX, Kaelin DL, Wall BE. Deep venous thrombo- sis: incidence on admission to a brain injury rehabilitation program. Arch Phys Med Rehabil 1996;77:11825.

22. Smeeth L, Cook C, Thomas S, Hall AJ, Hubbard R, Vallance P. Risk of deep vein thrombosis and pulmonary embolism after acute infection in a community setting. Lancet 2006;367:1075-9.

23. Clayton TC, Gaskin M, Meade TW. Recent respiratory infection and risk of venous thromboembolism: casecontrol study through a general practice database. Int J Epidemiol 2011;40:819-27.

24. Fujita Y, Nakatsuka H, Namba Y, Mitani S, Yoshitake $\mathrm{N}$, Sugimoto E, et al. The incidence of pulmonary embolism and deep vein thrombosis and their predictive risk factors after lower extremity arthroplasty: a retrospective analysis based on diagnosis using multidetector CT. J Anesth 2015;29:235-41.

25. Kimmell KT, Jahromi BS. Clinical factors associated with venous thromboembolism risk in patients undergoing craniotomy. J Neurosurg 2015;122:1004-11.

26. Kim JY, Khavanin N, Rambachan A, McCarthy RJ, Mlodinow AS, De Oliveria GS Jr, et al. Surgical duration and risk of venous thromboembolism. JAMA Surg 2015;150:110-7.

27. Legnani C, Palareti G, Cosmi B, Cini M, Tosetto A, Tripodi A, et al. Different cut-off values of quantitative D-dimer methods to predict the risk of venous thromboembolism recurrence: a post-hoc analysis of the PROLONG study. Haematologica 2008;93:900-7.

28. Pulivarthi S, Gurram MK. Effectiveness of d-dimer as a screening test for venous thromboembolism: an update. N Am J Med Sci 2014;6:491-9.

29. Hu X, Fang Y, Ye F, Lin S, Li H, You C, et al. Effects of plasma D-dimer levels on early mortality and longterm functional outcome after spontaneous intracerebral hemorrhage. J Clin Neurosci 2014;21:1364-7.

30. Stein J, Brandstater ME. Stroke rehabilitation. In: Frontera WR, DeLisa JA, editors. DeLisa's physical medicine \& rehabilitation: principles and practice. 5 th ed. Philadelphia: Lippincott Williams \& Wilkins; 2010. p. 551-74. 